\title{
Papel da atividade física na avaliação e tratamento da insuficiência cardíaca crônica
}

\author{
Salvador Serra ${ }^{1}$
}

\section{INTRODUÇÃO}

Estima-se que existam 15 milhões de pacientes com insuficiência cardíaca no mundo.

Definida como a incapacidade de o coração desenvolver um débito suficiente para atender às necessidades metabólicas dos tecidos periféricos, a insuficiência cardíaca é uma situação clínica grave e para a qual tende a evoluir a maioria das doenças cardíacas. O envelhecimento da população e um melhor controle, embora parcial, de diversas afecções cardiovasculares, tornam este processo praticamente inexorável e cada vez mais prevalente em muitos países, inclusive no Brasil.

Em sua forma crônica, a insuficiência cardíaca (ICC) manifesta-se com diversos sinais e sintomas, porém a sua característica clínica mais evidente é a incapacidade de sustentar esforços físicos progressivamente menores. Esta limitação restringe física, social e psicologicamente o paciente, além de constituir um balizador da gravidade da própria insuficiência cardíaca.

Por outro lado, as medições funcionais do coração durante o repouso efetivamente não refletem o grau de limitação que pode ocorrer com o aumento da demanda periférica durante o esforço. Além disto, sintomas habitualmente referidos pelos pacientes com ICC freqüentemente subestimam a verdadeira capacidade física, merecendo, portanto, sua avaliação objetiva ao exercício através do teste de esforço. Segundo a Sociedade Européia de Cardiologia, os pontos essenciais em relação à realização de teste ergométrico nos pacientes com insuficiência cardíaca são mostrados no quadro 1.

1. Mestre em Cardiologia pela UFF; Especialista em Cardiologia pela SBC/ AMB; Pós-graduado em Medicina do Exercício pela UFRJ; Chefe do Serviço de Ergometria e Reabilitação Cardíaca do Instituto Estadual de Cardiologia Aloysio de Castro-RJ; Co-responsável pelo Serviço de Ergometria do Hospital PróCardíaco-RJ.

Recebido em: 5/1/2001

Aceito em: 22/1/2001.

Endereço para correspondência:

Rua Paulino Fernandes, 25/802

22270-050 - Rio de Janeiro, RJ

E-mail: sserra@cremerj.com.br
Apesar de fornecer menor número de informações que o teste de esforço tradicional, recentemente têm sido utilizadas novas abordagens alternativas, e de custo mínimo, na avaliação funcional da insuficiência cardíaca. São eles: 1 . Teste dos Seis Minutos e 2. Shuttle Walk Test.

O Teste dos Seis Minutos visa quantificar a distância percorrida durante caminhada realizada no tempo estipulado em seis minutos. Grandes ensaios clínicos que visavam estudar o efeito de drogas no tratamento da ICC utilizaram este teste. Alguns estudos mostram correlação inversa entre a distância percorrida durante os seis minutos e a mortalidade.

Ainda mais recentemente foi desenvolvido o Shuttle Walk Test que, através de protocolo sonoro, orienta o paciente a caminhar uma distância de 10 metros em velocidades progressivamente mais rápidas. Este teste parece trazer melhores resultados na avaliação de pacientes com ICC quando comparado com o Teste de Seis Minutos.

Por outro lado, embora estes testes de caminhada, e principalmente os testes ergométricos clássicos, permitam obter informações funcionais e prognósticas importantes, a análise simultânea de gases, procedimento denominado ergoespirometria ou teste de esforço cardiopulmonar (TECP), diminui o componente subjetivo na identificação do momento da interrupção do esforço, além de, com fidelidade e reprodutibilidade, auxiliar na identificação da origem da dispnéia ao exercício, fornecer dados consistentes da funcionalidade cardiopulmonar e predizer o prognóstico dos pacientes em insuficiência cardíaca crônica.

\section{QUADRO 1}

Pontos essenciais para a realização de teste ergométrico na insuficiência cardíaca

1) Teste de esforço somente na insuficiência cardíaca estável

2) Medida direta do consumo de oxigênio é preferível aos valores estimados

3) Individualização dos protocolos (rampa, Naughton)

4) Estágios com incrementos de 1 MET são recomendados

5) Duração ótima do teste de 8 a 12 minutos

6) Teste de caminhada para testes submáximos 


\section{TESTE DE ESFORÇO CARDIOPULMONAR}

Poucas unidades ergométricas no Brasil dispõem dos equipamentos para a sua realização. Ao médico clínico, em particular ao cardiologista, limitado esclarecimento tem sido dado sobre o método e o seu significado na sua prática corrente. Somente poucos pacientes com ICC têm recebido a recomendação para se submeter à avaliação ao esforço, mesmo que seja dentro das possíveis limitações do teste ergométrico convencional. Dentro deste perfil, tornase interessante a abordagem do TECP na ICC em face da importância do método e as decisões que podem vir a ser tomadas com base na interpretação global dos seus resultados. Iremos, sumariamente, apontar pontos de maior interesse no TECP, destacando algumas variáveis avaliadas durante o procedimento, de especial importância na ICC.

\section{Protocolo de realização}

O paciente com insuficiência cardíaca, mesmo que clinicamente estável e apto a realizar o TECP, freqüentemente apresenta uma limitação imposta pela sua própria doença. Há, portanto, que informá-lo antecipadamente sobre algumas características do método. A colocação de máscara facial ou bocal, o impedimento mecânico da respiração nasal e a impossibilidade de comunicação verbal geralmente ocasionam, ainda durante o pré-teste, um grau de ansiedade e temor em relação ao exame, compreensiva e consideravelmente superior ao do indivíduo saudável ou atleta.

A escala de sensação subjetiva de cansaço (Borg) deve ser esclarecida, informando que as comunicações obviamente só poderão ser gestuais, que será interrompido o esforço somente no caso de alcançar o grau 10 da escala, embora, caso o paciente solicite, a utilização de gesto predeterminado interromperá o esforço e o teste como um todo. Esta orientação é altamente tranqüilizadora para o paciente e aumenta a segurança na realização de TECP.

Rotineiramente preconizamos a utilização dos protocolos individualizados de rampa em todas as situações, em particular durante o TECP na ICC. A adaptação em geral é excelente, embora um segundo exame possa permitir, após o aprendizado promovido com o primeiro, um desempenho mais satisfatório. Temos observado grande reprodução dos dados, excetuando o $\dot{\mathrm{VO}}_{2}$ de pico, que tende a ser mais elevado após um teste inicial.

\section{VंO $\mathrm{O}_{2}$ pico}

O consumo máximo de oxigênio ( $\mathrm{V}_{2}$ máx) é considerado o dado de maior expressão e de uso mais freqüente na avaliação funcional ao esforço de indivíduos saudáveis, doentes e atletas. $\mathrm{O} \dot{\mathrm{VO}}_{2}$ máx poderia ser definido como o consumo de oxigênio que, apesar do aumento da intensidade do esforço, se manteria constante ou com mínimas variações. Entretanto, como diversos sintomas podem limitar o paciente com ICC a alcançar o $\dot{V}_{2}$ máx, o momento limite da sustentação do esforço progressivo tem sido denominado de $\dot{\mathrm{VO}} 2$ pico. $\mathrm{O} \dot{\mathrm{V}} \mathrm{O}_{2}$ pico correlaciona-se diretamente com o débito cardíaco durante o esforço, sendo este o maior determinante da limitação da potência aeróbia nos pacientes assintomáticos ou moderadamente sintomáticos.

Ao contrário do teste ergométrico convencional, no TECP a interpretação das múltiplas variáveis e relações entre elas auxilia identificar se o paciente está realizando esforços efetivamente intensos. A utilização progressivamente maior da gordura, ao invés do carboidrato, como substrato energético durante o esforço, passa a ocorrer nas maiores intensidades, e, através da relação $\dot{\mathrm{V} C \mathrm{CO}_{2}} / \mathrm{V}_{2}$ (quociente respiratório) podemos inferir que o paciente encontra-se próximo do seu limite. Valores do quociente respiratório superiores a 1,0 (ou 1,1 para outros autores) são objetivamente sugestivos da proximidade do esforço máximo. Indivíduos com ICC, que durante o TECP ultrapassam o limiar anaeróbio (LA) [ou limiar ventilatório caso procuremos identificar o LA através do TECP], podem também ser considerados como tendo realizado esforço intenso e/ou próximo de seu máximo.

$\dot{\mathrm{VO}}_{2}$ pico $=$ Débito cardíaco do pico $X$ Diferença arteriovenosa de $\mathrm{O}_{2}$ do pico

Além de ser a referência mais utilizada na avaliação funcional na ICC, o $\mathrm{V}_{2}$ pico auxilia também na estratificação do prognóstico da cardiopatia, seja através da medida em valor absoluto, ou pelo percentual deste resultado em relação ao estimado para um dado paciente. Enquanto na ICC um $\dot{\mathrm{V}} \mathrm{O}_{2}$ pico superior a $20 \mathrm{ml} \mathrm{O}_{2} \cdot \mathrm{kg}^{-1} \cdot \mathrm{min}^{-1}$ sugere baixo índice de mortalidade para o ano seguinte, valores inferiores a $10 \mathrm{ml} \mathrm{O} \cdot \mathrm{kg}^{-1} \cdot \mathrm{min}^{-1}$ projetam uma mortalidade tão elevada quanto $80 \%$ também em um ano, o que torna o transplante cardíaco nestes casos uma possibilidade.

\section{Limiar anaeróbio}

Refletindo o início do aumento do acúmulo de lactato sanguíneo, o limiar anaeróbio é uma importante informação obtida em intensidade submáxima de esforço. Ele, de maneira semelhante ao $\dot{\mathrm{V}} \mathrm{O}_{2}$ pico, é utilizado na avaliação funcional de indivíduos saudáveis, atletas e na ICC.

Em concordância com a literatura, temos observado que o limiar ventilatório medido em $\mathrm{ml} \mathrm{O} \cdot \mathrm{kg}^{-1} \cdot \mathrm{min}^{-1}$, é, em média, bem inferior ao do indivíduo saudável, porém, ao inverso deste, no portador de ICC o valor percentual do LA em relação ao $\mathrm{V}_{2}$ pico, é, em média, mais elevado. Este percentual mais alto do $\dot{\mathrm{VO}}_{2}$ do LA em relação ao $\dot{\mathrm{V}} \mathrm{O}_{2}$ pico na ICC pode ser atribuído à precoce interrupção do esforço 
devido aos sintomas, freqüentemente limitantes. Assim, o $\dot{\mathrm{V}} \mathrm{O}_{2}$ do LA aproxima-se do momento do término do esforço ( $\dot{\mathrm{VO}}_{2}$ pico). Os valores absolutos do $\dot{\mathrm{VO}}_{2}$ pico e do $\dot{\mathrm{VO}}_{2}$ do LA são menores quando comparados com os de indivíduos saudáveis.

\section{Equivalentes ventilatórios}

As relações da ventilação pulmonar (VE - medida em litros por minuto) com o $\dot{\mathrm{VO}}_{2}\left(\mathrm{VE} / \mathrm{V}_{2}\right)$ e de $\mathrm{VE}$ com o $\dot{\mathrm{V} C O}{ }_{2}\left(\mathrm{VE} / \mathrm{VCO}_{2}\right)$, são denominadas de equivalentes ventilatórios de $\mathrm{O}_{2}$ e $\mathrm{CO}_{2}$, respectivamente.

$\mathrm{O}$ equivalente ventilatório de $\mathrm{O}_{2}$ auxilia na avaliação funcional ventilatória durante o esforço. Há necessidade de aproximadamente 30 litros de VE para consumir um litro de $\mathrm{O}_{2}$ Valores elevados desta relação $\left(\mathrm{VE} / \mathrm{VO}_{2}\right)$, desde que afastadas situações de ordem emocional que levem ao aumento desproporcional da ventilação, expressam ineficiência funcional ventilatória. Após apresentar uma queda no começo do esforço, o momento inicial da elevação da curva do equivalente ventilatório de $\mathrm{O}_{2}$ tem sido utilizado na identificação do limiar ventilatório.

Expressando indiretamente a relação entre a ventilação e a perfusão e, conseqüentemente, avaliando o espaço morto durante o exercício, o equivalente ventilatório de $\mathrm{CO}_{2}$ temse mostrado importante na estratificação do prognóstico na insuficiência cardíaca. Estudando pacientes com ICC, MacGowan et al., medindo o $\mathrm{VE} / \mathrm{VCO}_{2}$ no limiar ventilatório, mostraram que pacientes com valor desta relação superior a 50, quando associado a $\mathrm{V}_{2}$ de pico inferior a $15 \mathrm{ml}$ $\mathrm{O}_{2} \cdot \mathrm{kg}^{-1} \cdot \mathrm{min}^{-1}$, evoluíram, durante os 3,8 anos de seguimento, com $82 \%$ de mortalidade, contra $22 \%$ naqueles com $\mathrm{VE} / \mathrm{VCO}_{2}<50$ e $\dot{\mathrm{VO}}{ }_{2}$ de pico superior a $15 \mathrm{ml} \mathrm{O}_{2} \cdot \mathrm{kg}^{-1} \cdot \mathrm{min}^{-1}$.

Outros mecanismos tentam explicar a associação de valor elevado do $\mathrm{VE} / \mathrm{VCCO}_{2}$ com pior evolução da ICC. Um deles refere-se à menor produção e/ou liberação de óxido nítrico endotelial (ON) durante o esforço na insuficiência cardíaca grave. Menor quantidade de ON implicaria menor vasodilatação, inclusive do leito vascular pulmonar, levando, consequientemente, a um aumento compensatório da ventilação e uma elevação da relação $\mathrm{VE} / \mathrm{VCO}_{2}$.

\section{ATIVIDADE FÍSICA NA INSUFICIÊNCIA CARDÍACA}

Vários mecanismos interferem na tolerância ao esforço na insuficiência cardíaca crônica: 1) hemodinâmica central (débito cardíaco), 2) atividade neuro-hormonal (atividade simpática, sensibilidade beta-adrenérgica, equilíbrio de sistemas vasoativos e natriuréticos e antinatriuréticos), 3) resposta periférica (principalmente musculatura esquelética) e 4) função pulmonar.
Alguns autores atribuem um papel importante da musculatura esquelética na responsabilidade da limitação ao esforço destes pacientes, acreditando existir uma verdadeira miopatia esquelética, com modificações estruturais e funcionais do miócito, em grande parte atribuídas ao descondicionamento físico. Outros autores, entretanto, contestam parcialmente esta interpretação, argumentando que semelhantes modificações são encontradas também na musculatura respiratória.

Discordâncias à parte, a multiplicidade de fatores envolvidos na limitação da tolerância na ICC parece ser influenciada pela prática regular de atividade física. Pacientes com ICC participantes de programas de reabilitação, nos quais são aplicados exercícios físicos regularmente, freqüentemente registram efeitos favoráveis no $\mathrm{V}_{2}$ de pico, na função respiratória, no limiar anaeróbio, nas variáveis hemodinâmicas, no aspecto psicológico, na qualidade de vida, na morbidade e mortalidade (figuras 1-3).

Progressivamente, os serviços de reabilitação têm incluído cada vez mais um quantitativo maior desses pacientes em seus programas. Raramente presentes há uma década, atualmente eles já constituem cerca de 25 a $30 \%$ dos pacientes em reabilitação do Instituto Estadual de Cardiologia Aloysio de Castro, no Rio de Janeiro. Obviamente, a situação de estabilidade clínica é pré-requisito para a inclusão do paciente; entretanto, na dependência da estrutura dos serviços, a conduta quanto à participação de pacientes, inclusive em classe funcional grau III, poderá tornarse menos limitada.

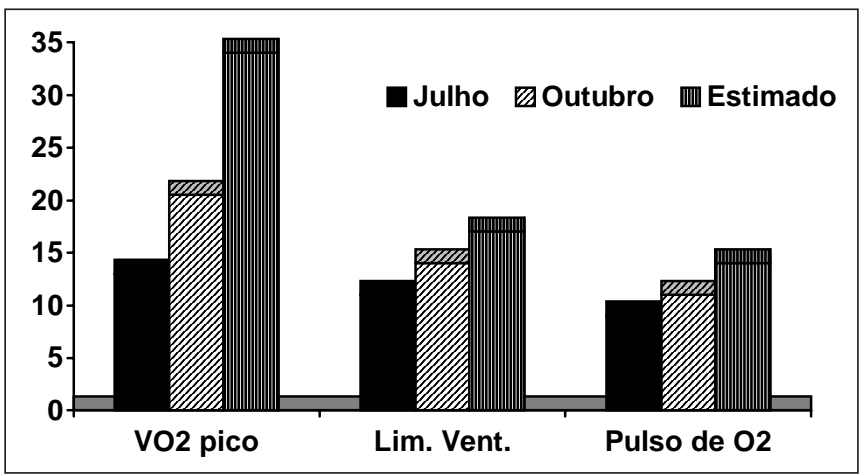

Fig. 1 - Paciente 1. Respostas metabólicas de programa de reabilitação em portador de insuficiência cardíaca por cardiomiopatia isquêmica. O paciente de 62 anos havia apresentado grave quadro de infarto agudo do miocárdio, era hipertenso, portador de síndrome plurimetabólica, apresentava sobrepeso e era tabagista. A fração de ejeção ventricular esquerda era de $22 \%$. Nota-se, após quatro meses de programa, que, apesar de persistirem valores inferiores ao estimado em relação ao indivíduo saudável, há um nítido aumento do consumo de oxigênio do pico do esforço e do limiar ventilatório, e uma elevação do pulso de oxigênio, variável esta diretamente relacionada ao volume sistólico (avaliações pré e pós-programa realizadas através de teste de esforço cardiopulmonar). 


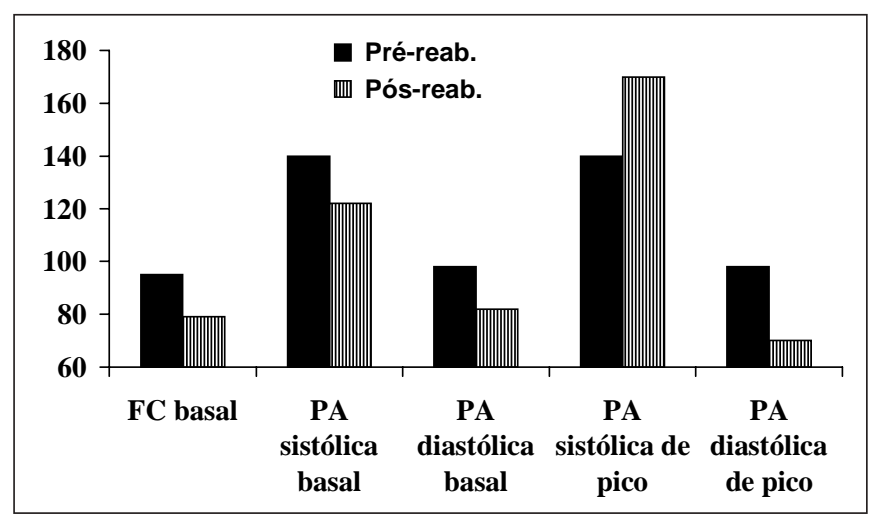

Fig. 2 - Paciente 2. Masculino, 57 anos, em insuficiência cardíaca, classe funcional grau III decorrente de cardiomiopatia isquêmica, fração de ejeção ventricular esquerda: $30 \%$. Freqüência cardíaca e pressão arterial pré e intra-esforço na avaliação inicial controle e após um ano de reabilitação. Observa-se redução da freqüência cardíaca e da pressão arterial (PA) sistólica e diastólica em repouso. No pico do esforço, o aumento da PA sistólica no teste realizado ao término do programa pode ser atribuído ao aumento da tolerância e ao melhor desempenho funcional cardiovascular, também expressado pela queda da resistência vascular periférica inferida através da acentuação da redução da PA diastólica.

Poucos estudos efetivamente mostram experiência na aplicação de atividade física em pacientes com ICC classe funcional IV. Acreditamos, entretanto, que talvez, ou mesmo principalmente, os resultados de programas individualizados possam ser ainda mais favoráveis neste subgrupo de pacientes estáveis. Parte desta presunção pode ser admitida considerando os efeitos positivos da atividade física como algo semelhantes àqueles obtidos com betabloqueadores. Os resultados recentes do estudo Copernicus, mostrando o efeito favorável do Carvedilol também na ICC classe funcional IV, nos permite aventar aquela possibilidade.

\section{CONCLUSÃO}

A abordagem ao paciente em insuficiência cardíaca crônica, mais do que a maioria das demais condições clínicas, passa atualmente por procedimentos que utilizam formas de tratamento, farmacológico ou não, que, há apenas poucas décadas, seriam consideradas como contra-indicações absolutas.

Temos muito que aprender com a síndrome multifacetada da insuficiência cardíaca. Entretanto, todos os indicati-

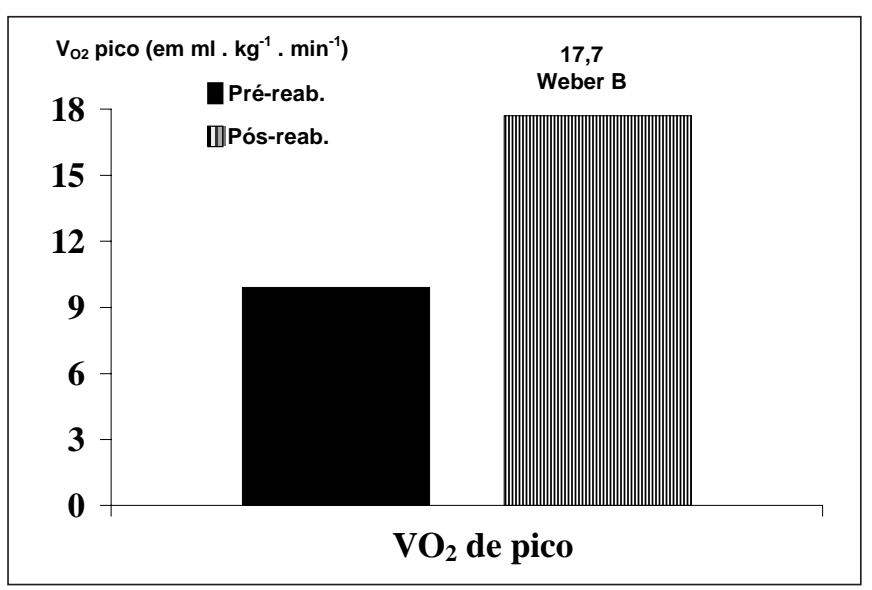

Fig. 3 - Paciente 2, referido na figura 2. Consumo de oxigênio no pico do esforço $\left(\dot{V}_{2}\right.$ pico $)$ pré e pós-programa de reabilitação. $O$ aumento do $\dot{V} \mathrm{O}_{2}$ pico na avaliação após um ano de reabilitação permite inserir o paciente em melhor classe funcional que, segundo Weber (classificou funcionalmente de $A$ a $D$ ), está associada a importante redução no índice de mortalidade em um ano (classe D: $80 \%$; classe B: <20\%).

vos apontam para um papel de destaque da atividade física, seja na avaliação funcional e prognóstica, como também na sua ativa e importante participação no tratamento.

\section{BIBLIOGRAFIA RECOMENDADA}

1. Shah NB, Massie BM. Epidemiology of heart failure: evolving trends. In: Balady GJ, Piña IL. Exercise and heart failure. Armonk: Futura Publishing, 1997:3.

2. Recommendations for exercise testing in chronic heart failure patients. Working group report. Eur Heart J 2001;22:37-45.

3. Witte K, Thackray S, Clark AL, Cooklin M, Cleland JGF. Clinical trials update: Improvement-HF, Copernicus, Mustic, Aspect-II, Apricot and Heart. Eur Heart J 2000;2:455-60.

4. MacGowan GA, Janosko K, Cechetti A. Exercise-related ventilatory abnormalities and survival in congestive heart failure. Am J Cardiol 1997; 79:1264-6.

5. Sullivan M, Duscha BD. Cardiovascular adaptations to exercise training in left ventricular dysfunction. In: Balady GJ, Piña IL. Exercise and heart failure. Armonk: Futura Publishing, 1997:261.

6. Cohen-Solal A. Cardiopulmonary exercise testing in chronic heart disease. In: Wasserman K. Exercise gas exchange in heart disease. Armonk: Futura Publishing, 1996:17.

7. Chua TP, Ponikowski P, Harrington D. Clinical correlates and prognosis significance of the ventilatory response to exercise in chronic heart failure. J Am Coll Cardiol 1997;29:1585-90.

8. Morales FJ, Montemayor T, Martinez A. Shuttle versus six-minute walk test in the prediction of outcome in chronic heart failure. Intern J Cardiol 2000;76:101-5. 